$\Rightarrow$ UROLOGIC ONCOLOGY

\section{New standard for localized UTUC}

Upper tract urothelial carcinoma (UTUC) has a worse stage-for-stage prognosis than the related disease urothelial carcinoma of the bladder. UTUC is muscle-invasive or locally advanced at diagnosis in $>50 \%$ of patients and a similar proportion ultimately die of the disease. Drawing on the clinical benefits achieved with platinum-based chemotherapy for bladder cancer, Birtle et al. initiated the phase III POUT trial of adjuvant chemotherapy for patients with muscleinvasive or lymph-node-positive UTUC. Now, data from this trial support this approach as a new standard of care.

Enrolment for the POUT trial was stopped early after a preplanned interim analysis of data from 261 patients revealed a disease-free survival (DFS) benefit of chemotherapy following nephroureterectomy and resection of any known abnormal lymph nodes. Among 131 patients who received up to four cycles of gemcitabine plus carboplatin or cisplatin, estimated 3-year DFS was $71 \%$, compared with $46 \%$ in 129 patients who underwent standard surveillance only (HR 0.45, 95\% Cl 0.30-0.68; $P=0.0001)$; 3-year metastasis-free survival was also improved $(71 \%$ versus $53 \%$; $\mathrm{HR} 0.48,95 \% \mathrm{Cl}$ $0.31-0.74 ; P=0.0007)$. These benefits were largely unchanged in multivariate analyses.

Unsurprisingly, chemotherapy significantly increased the risk of toxicities: grade $>3$ adverse events, mostly decreased neutrophil and/or platelet counts, nausea and vomiting or febrile neutropenia, occurred in $44 \%$ of patients, compared with only $4 \%$ of the surveillance group $(P<0.0001)$. Importantly, no treatment-related deaths occurred. Quality of life questionnaires were completed by at least $70 \%$ of patients in each group and revealed declines in mean overall global health status scores during and immediately after chemotherapy, which resolved by 12 months.

UTUC is a rare disease affecting $\sim 2$ people per 100,000 . Thus, Birtle et al. should be commended for performing what is almost certainly the first dedicated phase III study of perioperative therapy for UTUC and the largest-cohort randomized controlled trial in this disease to date. Longer-term results of POUT, including overall survival data, are awaited.

David Killock

This article is modified from the original in Nat. Rev. Clin. Onc. (https://doi.org/10.1038/s41571-020-0354-6).

ORIGINAL ARTICLE Birtle, A. et al. Adjuvant chemotherapy in upper tract urothelial carcinoma (the POUT trial): a phase 3 , open-label, randomised controlled trial. Lancet https://doi.org/ 10.1016/S0140-6736(20)30415-3 (2020)

\title{
STONES
}

\section{Comparing outcomes of SMP and Miniperc}

A new international multicentre retrospective study shows that use of super-mini percutaneous nephrolithotomy (SMP) is beneficial over Miniperc in patients with renal calculi of $2-3 \mathrm{~cm}$ in diameter.

In total, 3,525 patients underwent treatment, of whom 2,012 received SMP and 1,513 received Miniperc. The treatment groups were comparable for mean BMI, stone density, positive preoperative urine culture rate and mean STONE score but required additional matching for sex ratio, mean age, rate of STONE score categories and hydronephrosis level. Further analysis was conducted using 1,380 matched patients from each group. Stone-free rate (SFR; primary outcome), blood loss, operating time, postoperative pain measured using a visual analogue scale (VAS; range 1-10), auxiliary procedures, complications, tubeless rate and length of hospital stay (all secondary outcomes) were evaluated.

Overall, no statistical difference in final or initial SFR was observed between the groups. However, SMP resulted in significantly lower pain (VAS score

\section{INCONTINENCE}

\section{TVT-O for SUI}

Tension-free vaginal tape-obturator (TVT-O) for the treatment of female stress urinary incontinence (SUI) is safe and highly effective, according to results published in Neurourology and Urodynamics.

In an international multicentre study, 168 women underwent TVT-O implantation. Inclusion criteria were self-reported pure SUl symptoms in addition to urodynamically proven urodynamic stress incontinence (USI). After assessment at 1,5 and 10 years, the authors report the most recent follow-up data of 157 evaluable patients at 13 years.

Both subjective and objective outcomes were assessed. At 13 years after surgery, 150 (95\%) patients reported themselves cured, based on their responses to the International Consultation on Incontinence Questionnaire Short Form (ICIQ-SF), the Patient Global Impression of Improvement (PGI-I) scale and a patient-satisfaction scale. Objective cure, defined as the absence of urine leakage during a full-bladder stress test in the lithotomy and upright positions, was reported for 141 (90\%) women.
4.7 versus 5.1, $P<0.001)$, shorter hospital stay ( 3.1 versus 4.8 days, $P<0.001$ ), less blood loss (haemoglobin drop 12.5 versus $13.7 \mathrm{~g} / \mathrm{l}, P=0.015)$ and higher tubeless rate (72.6 versus $57.8 \%, P<0.001$ ) than Miniperc, which also had a significantly higher rate for one complication (fever; 12.0 versus $8.4 \%$, $P=0.002$ ). Operative times and auxiliary procedure ratio were comparable between the treatments.

Classification of patients into groups with stone diameters of $2-3 \mathrm{~cm}, 3-4 \mathrm{~cm}$ and $>4 \mathrm{~cm}$ revealed that for stones $>4 \mathrm{~cm}$, SMP resulted in significantly lower SFR and longer operating times, which were comparable in both of the remaining groups.

On the basis of these findings, the authors propose SMP as a safer and more efficacious treatment than Miniperc for renal calculi $2-3 \mathrm{~cm}$ in diameter and suggest that SMP could be indicated for the treatment of larger renal calculi up to $4 \mathrm{~cm}$ in diameter.

Tim Thomas

ORIGINAL ARTICLE Liu, Y. et al. Management of large renal stones in super-mini-percutaneous nephrolithotomy (SMP): an international multicenter comparative study. BJU Int. https://doi.org/10.1111/bju.15066 (2020)
Using the $\chi^{2}$ test for trend, Serati et al. report that time elapsed since treatment is not significantly associated with a decrease in cure rate for either subjective $(P=0.78)$ or objective $(P=0.10)$ cure. The cure rates at 1,5 and 10 years were $95 \%, 95 \%$ and $97 \%$ for subjective cure and $95 \%$, $91 \%$ and $92 \%$ for objective cure, respectively. Factors that potentially contributed to recurrent subjective and objective USI (and, therefore, subjective and objective treatment failure) during the study period were also assessed: of the 14 variables assessed, obesity and previous antiincontinence procedures each predicted subjective $(P=0.03$ and $P=0.009$, respectively) and objective $(P=0.04$ and $P=0.01)$ failure of TVT-O.

In addition, the study reports a significantly higher risk of sling exposure after 10 years following treatment than before 10 years $(P=0.05)$, although the rate of exposure observed was low and sling exposure occurred in 4 of 157 (2.5\%) women.

Tim Thomas

ORIGINAL ARTICLE Serati, M. et al. TVT-O for treatment of pure urodynamic stress urinary incontinence: efficacy and adverse effects at 13-years follow-up. Neurourol. Urodyn. https://doi.org/10.1002/nau.24358 (2020) 